\title{
Lesão por pressão relacionada a dispositivos médicos: prevenção e fatores de risco associados
}

RESUMO | Introdução: A lesão por pressão é encontrada na pele ou tecido subjacente, geralmente sobre uma proeminência óssea, resultante da exposição à pressão ou forças de cisalhamento, possuindo fatores intrínsecos e extrínsecos: imobilidade, inconsciência, perda da continência urinária e fecal, deficiência nutricional, doenças crônico degenerativas, peso e relacionados a dispositivos médicos. Objetivo: Descrever a prevenção e fatores de risco para Lesão por pressão relacionadas à dispositivos médicos. Método: Revisão integrativa de literatura, entre 2010 a 2020 nas bases de dados LILACS, PubMed, Bdenf e site de busca Scielo. Resultados: Foram incluídos nove estudos, sendo: quatro $(44,4 \%)$ publicações que descrevem os principais dispositivos relacionados a estas lesões. Conclusão: Foi descrito os fatores associados ao desenvolvimento de Lesões por Pressão Relacionadas a Dispositivos Médicos e como preveni-las, identificando quais os dispositivos de risco, e medidas de prevenção e tratamento, cuidados específicos e eficazes por meio dos profissionais de enfermagem na prevenção e tratamento.

Descritores: Lesão por Pressão; Equipamentos e provisões; Enfermagem.

ABSTRACT | Introduction: Pressure injury is found in the skin or underlying tissue, usually over a bony prominence, resulting from exposure to pressure or shear forces, having intrinsic and extrinsic factors: immobility, unconsciousness, loss of urinary and fecal continence, nutritional deficiency, chronic degenerative diseases, weight and related to medical devices. Objective: To describe the prevention and risk factors for Pressure Injury related to medical devices. Method: Integrative literature review, between 2010 and 2020 in LILACS, PubMed, Bdenf and Scielo search engine databases. Results: Nine studies were included: four (44,4\%) publications describing the main devices related to these injuries. Conclusion: The factors associated with the development of Pressure Injuries Related to Medical Devices and how to prevent them were described, identifying which are the risk devices, and prevention and treatment measures, specific and effective care through nursing professionals in prevention and treatment. Keywords: Pressure injury; Equipment and supplies; Nursing.

RESUMEN | Introducción: La lesión por presión se encuentra en la piel o tejido subyacente, generalmente sobre una prominencia ósea, como resultado de la exposición a fuerzas de presión o cizallamiento, teniendo factores intrínsecos y extrínsecos: inmovilidad, inconsciencia, pérdida de continencia urinaria y fecal, deficiencia nutricional, crónico degenerativo. enfermedades, peso y relacionados con dispositivos médicos. Objetivo: Describir la prevención y los factores de riesgo de las lesiones por presión relacionadas con los dispositivos médicos. Método: Revisión integrativa de la literatura, entre 2010 y 2020 en las bases de datos de los motores de búsqueda LILACS, PubMed, Bdenf y Scielo. Resultados: Se incluyeron nueve estudios: cuatro (44,4\%) publicaciones que describen los principales dispositivos relacionados con estas lesiones. Conclusión: Se describieron los factores asociados al desarrollo de Lesiones por Presión Relacionadas con Dispositivos Médicos y cómo prevenirlas, identificando cuáles son los dispositivos de riesgo, y las medidas de prevención y tratamiento, atención específica y efectiva a través de profesionales de enfermería en prevención y tratamiento.

Palabras claves: Lesión por presión; Equipo y suministros; Enfermería.

Carla Nascimento Souza Santos

Discente do curso de Graduação em Enfermagem das Faculdades Integradas de Três Lagoas - AEMS.

ORCID: 0000-0002-3991-8527.

\section{Gabriela Maia de Oliveira}

Discente do curso de Graduação em Enfermagem das Faculdades Integradas de Três Lagoas - AEMS.

ORCID: 0000-0003-1163-1249.

\section{Flávia Carla Takaki Cavichioli}

Enfermeira. Mestre em Ciência, Tecnologia e Gestão Aplicadas a Regeneração Tecidual pela UNIFESP.

ORCID-ID: 0000-0003-2277-9548.

Hélio Martins do Nascimento Filho

Enfermeiro. Mestre em Ciência, Tecnologia e Gestão Aplicadas à Regeneração Tecidual pela Unifesp. Enfermeiro na Unidade Saúde da Família / USF em Conselheiro Lafaiete - MG. Professor na Pós-graduação em Enfermagem Dermatológica com Ênfase no Tratamento de Feridas na Faculdade FAIPE (Cuiabá-MT).

ORCID-ID: 0000-0003-3700-3910.

\section{Fabíola Arantes Ferreira}

Enfermeira. Mestre em Enfermagem em Terapia Intensiva, possui graduação em Enfermagem pela UEMG.

ORCID: 0000-0001-7360-820X.

\section{Daniela Tinti Moreira Borges}

Enfermeira. Mestre Profissional em Ciências pela UNIFESP/SP; Estomaterapeuta, pela FAMERP/SP (2019). Docente de Graduação em Enfermagem na AEMS (Faculdades Integradas de Três Lagoas/MS). ORCID: 0000-0001-8890-8918.
Recebido em: 26/07/2021

Aprovado em: 06/08/2021

INTRODUÇÃO

A s lesões por pressão (LP) são agravantes para a saúde pública, e indicam a eficácia do cuidado da saúde. O surgimento das lesões provoca um desequilíbrio para as pessoas acometidas e também para os cuidadores, sendo assim, altera o bem estar de todos os que estão envolvidos, é um problema relevante, podendo levar à morte. Conhecer o processo de evolução da LP é um fator fundamental para que as lesões não se desenvolvam. Cerca de 95\% das lesões podem ser evitadas, por meio do descobrimento prévio da intensidade da gravi- 
dade, executando cuidados com qualidade, fator esse que contribui grandemente para a prevenção ${ }^{(1)}$.

As (LPP) têm se intensificado nos últimos anos devido à maior perspectiva de vida da população, resultante da evolução na assistência à saúde, que tornou plausível a sobrevida de pacientes com doenças graves e anteriormente letais, transformadas em doenças crônicas e lentamente debilitantes. Essa alteração de perfil possibilitou na prática um gradativo número de pessoas com lesões cutâneas, basicamente a $\mathrm{LP}^{(2)}$.

Um aspecto relevante na assistência de Enfermagem é a preservação da integridade da pele, sendo primordial o uso de ações preventivas para a realização deste objetivo. Compreende-se como lesão por pressão aquela que se encontra na pele ou tecido subjacente, geralmente sobre uma proeminência óssea, resultante da exposição à pressão ou forças de cisalhamento, possuindo fatores intrínsecos e extrínsecos, como a imobilidade, inconsciência, perda da continência urinária e fecal, deficiência nutricional, doenças crônico degenerativas, peso e outros fatores. Representam-se essas lesões uma falha na saúde pública, e acabam acarretando o prolongamento do tempo de hospitalização, logo gerando impactos negativos nas despesas financeiras das instituiçõos de saúde ${ }^{(3)}$.

O National Pressure Injury Advisory Panel (NPIAP) ${ }^{(4)}$ redefiniu o sistema de teste para LP, abrangendo as LP associada ao dispositivo médico (LPRDM), estabelecidas como resultantes do uso de dispositivos programado e executado para fins diagnósticos ou terapêuti$\cos ^{(5)}$.

Habitualmente os dispositivos médicos são usados em ambientes hospitalares. Com a aquosidade e o aquecimento entre a pele e o dispositivo, ocorre a mudança do microclima da pele. Para uma vedação apropriada, é necessária na maioria das vezes a fi- xação firme destes dispositivos, criando uma pressão em áreas não usuais, em vez das proeminências ósseas, ou seja, o próprio dispositivo cria pressão. As fitas, correias entre outros materiais utilizados para a fixação dos dispositivos dificultam a inspeção da pele subjacente abaixo deles, elevando os riscos do aparecimento das lesões por pressão ${ }^{(6)}$.

Alguns dispositivos médicos como (tubos endotraqueais, máscaras de oxigênio, cateteres urinários, colares cervicais, tubos de traqueostomia / laços, meias de compressão, e a sonda nasogástrica) são encontrados entre as organizações de cuidados. As lesões por pressão (LPs) formadas a partir do uso dos dispositivos médicos constitui um evento clínico que requer atenção dos profissionais de saúde, pois integram em parte os cuidados prestados a pessoas em todos os serviços de saúde ${ }^{(7)}$.

A prevenção da LP caracteriza um cuidado imprescindível e requer muita atenção, pois o não aparecimento da lesão e o seu desenvolvimento, demanda menos trabalho da equipe em comparação ao tempo e gastos que são indispensáveis para resolver as implicações de seu aparecimento. A escassez em intervenções preventivas resulta com o progresso da LP, com base nessa veracidade, é crucial elaborar um plano de cuidado iminente e efetivo capaz de diminuir os efeitos nocivos da lesão, contribuindo assim para uma recuperação eficiente mais rápida do paciente ${ }^{(8)}$.

O objetivo do presente trabalho é descrever prevenção e fatores associados ao desenvolvimento de Lesões por Pressão Relacionadas a Dispositivos Médicos.

\section{MÉTODOS}

Trata-se de uma revisão integrativa de literatura, realizada seguindo os seis passos recomendados para este tipo de estudo: elaboração da questão norteadora "Quais são os fatores associados 


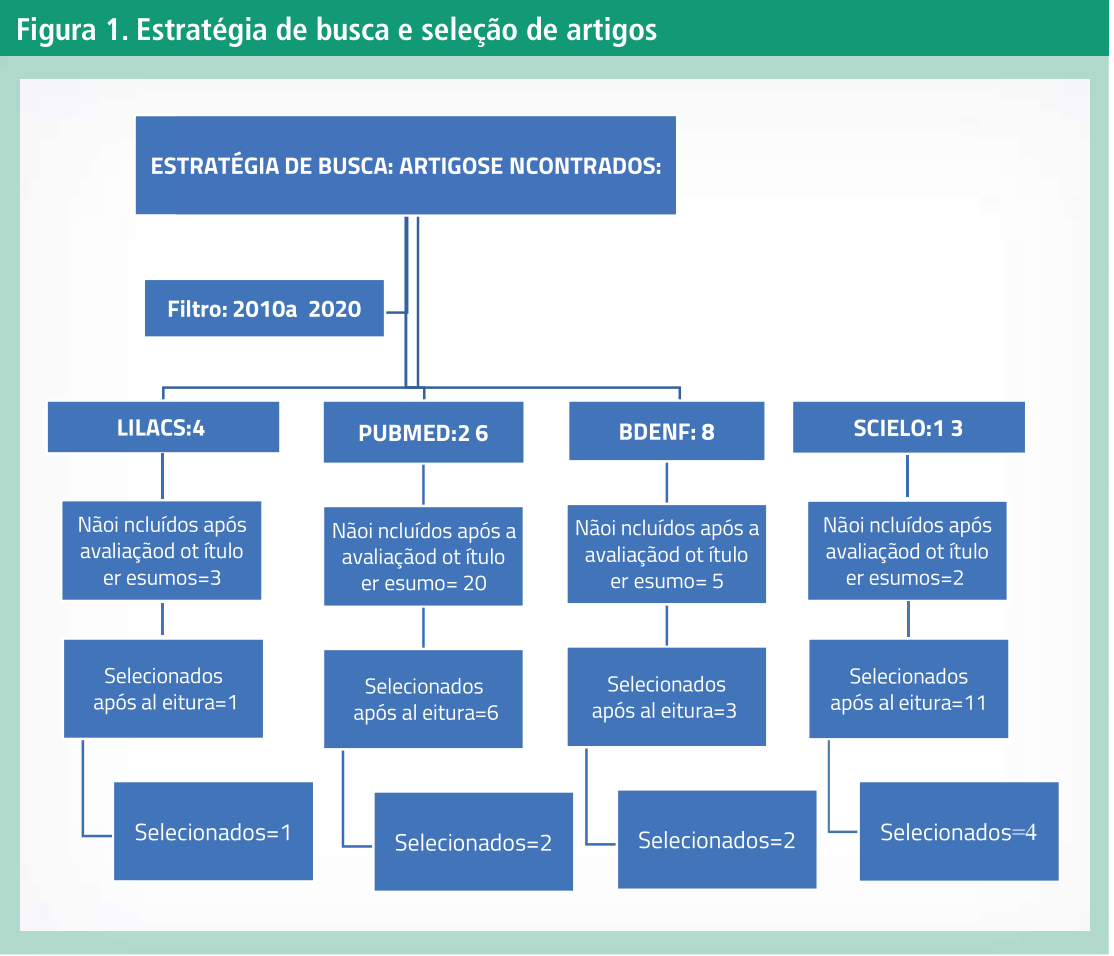

Fonte: Autores, 2020

ao desenvolvimento de LP relacionadas a dispositivos médicos e qual seu manejo clínico"? Busca na literatura, coleta de dados, análise crítica dos estudos incluídos, discussão e resultados e apresentação da revisão integrativa.

A busca bibliográfica ocorreu no período de março a maio de 2020 nas bases de dados MedLine, Lilacs, Bdenf, PubMed e site de busca Scielo. Foram utilizados os seguintes Descritores em Ciências da Saúde: Lesão por Pressão; Equipamentos e provisões; Enfermagem.

Os critérios de inclusão adotados foram artigos publicados entre 2010 e 2020; disponíveis na íntegra, nos idiomas português, inglês e espanhol; artigos com conteúdo apropriado para a proposta dessa pesquisa. Foram ex- cluídas as duplicidades, aqueles não coerentes com a temática pesquisada e os que não compreendem o período de registro determinado, não condizente assim com os objetivos do presente trabalho.

De acordo com os critérios estabelecidos, foram incluídos nove artigos, sendo um no Lilacs, dois no Bdenf, quatro no Scielo, dois na Pubmed e um documento.

\section{RESULTADOS}

Foram selecionados nove estudos, de modo que quatro $(44,4 \%)$ artigos descrevem os dispositivos médicos que causam lesões com maior frequência, sendo que o tubo orotraqueal é o dispositivo relacionado à maior incidência das LPRDM, especialmente atrás das orelhas. Dois $(22,2 \%)$ retratam as medidas preventivas; uma $(11,1 \%)$ publicação traz a atualização dos conceitos em lesão por pressão, um $(11,1 \%)$ trabalho verificou o conhecimento dos profissionais acerca da prevenção e tratamento destas feridas e um $(11,1 \%)$ descreve as condições do paciente associadas ao desenvolvimento das LPRDM. Os artigos incluídos neste estudo podem ser observados no Quadro 1.

É importante destacar que ações

\section{Quadro 1 - Artigos incluídos no estudo}

$\begin{array}{cccc}\begin{array}{c}\text { Base de } \\ \text { dados }\end{array} & \begin{array}{c}\text { Autores } \\ \text { e Ano de } \\ \text { publicação }\end{array} & \text { Título do artigo } & \text { Principais resultados } \\ \text { LILACS } & \begin{array}{c}\text { Correia e San- } \\ \text { tos, 2019(8) }\end{array} & \text { Lesão por Pressão: Medidas Terapêuticas Utilizadas } & \text { Analisando os cuidados com a LP, ressaltaram ações preventivas, } \\ & \text { por Profissionais de Enfermagem } & \text { visando evitar o agravamento das lesões e prevenir o surgimento de } \\ \text { novas LPs, com ações de limpeza da ferida e de terapia tópica. }\end{array}$

Bdenf Moraes et al. Conceito e Classificação de Lesão por Pressão: Atua2016(2) lização do National Pressure Ulcer Advisory Panel
Conforme estabelecido pelo NPIAP, o conceito de LPP é um dano localizado na pele e/ou tecido mole subjacente geralmente sobre proeminência óssea ou pode ainda estar relacionado a equipamentos médicos ou outro tipo de dispositivo. 0 uso correto do conceito e terminologia em LPP faz com que os profissionais possam avaliar e desenvolver estratégias de enfrentamento de maneira mais efetiva. 


\begin{tabular}{|c|c|c|c|}
\hline Bdenf & $\begin{array}{l}\text { Santin Júnior } \\
\text { et al., 2019(3) }\end{array}$ & $\begin{array}{l}\text { Educação Permanente: Ferramenta de Aprimoramen- } \\
\text { to Assistencial às Lesões por Pressão }\end{array}$ & $\begin{array}{l}\text { 90\% da equipe participante do estudo obteve uma média abaixo } \\
\text { do esperado em relação ao conhecimento referente à classificação } \\
\text { normativa das lesões por pressão, reforçando a necessidade da } \\
\text { educação permanente da equipe de saúde. }\end{array}$ \\
\hline
\end{tabular}

Os pacientes com dispositivos médicos eram significativamente mais propensos a desenvolver uma úlcera de pressão dispositivo. Ao utili-

$\begin{array}{cc}\text { PubMed } & \text { Black et al., } \\ 2010(6) & \text { Medical device related pressure ulcers in hospitalized } \\ \text { patients }\end{array}$
zar o dispositivo, os pacientes eram de 2 a 4 vezes mais propensos a desenvolver uma lesão por pressão. As lesões por pressão ocorreram com maior frequência nos lábios mãos e região occipital, relacionadas aos seguintes dispositivos médicos: tubo endotraqueal, cateter venoso e colar cervical

$\begin{array}{cccc}\text { PubMed } & \begin{array}{c}\text { Kayser et al., } \\ 2018(7)\end{array} & \begin{array}{c}\text { Prevalence and Analysis of Medical Device-Related } \\ \text { Pressure Injuries: Results from the International } \\ \text { Pressure Ulcer Prevalence Survey }\end{array} & \begin{array}{c}\text { A prevalência de LPRDM foi maior em hospitais de cuidados inten- } \\ \text { sivos de longo prazo do que em lugares de tratamento de pouca } \\ \text { intensidade e com prazo menor de hospitalização }\end{array}\end{array}$

Scielo Galleto et al., $\quad$ Lesões por Pressão Relacionadas a Dispositivos
2018(9) $\quad$ Médicos: revisão integrativa da literatura
A região cervical posterior e o nariz são os locais mais frequentemente acometidos pelas LPRDM. Foram identificados onze dispositivos de risco para o desenvolvimento das lesões, destacaram-se as máscaras de Ventilação Não Invasiva e o tubo orotraqueal, por ocasionarem lesão em seis diferentes locais do corpo. No que tange às medidas de prevenção e de tratamento das lesões, existem as gerais e as específicas ao tipo de dispositivo utilizado, sendo a maioria de simples execução e pautada principalmente na avaliação periódica da pele sob os dispositivos.

$\begin{array}{cccc}\text { Scielo } & \begin{array}{c}\text { Souza e Prado, A utilização Da Escala De Braden na UTI para preven- } \\ 2016(10)\end{array} & \begin{array}{c}\text { O número de publicações encontradas, apresentam uma trajetória } \\ \text { contínua ao longo dos anos. A prevenção da LP é uma preocupação } \\ \text { antiga e atual confirmando a elevada incidência desta complicação } \\ \text { principalmente em pacientes críticos. }\end{array}\end{array}$

0 dispositivo que mais desenvolveu LPRDM foi o tubo orotraqueal, com $63,76 \%$, e as orelhas foram o principal local, com $32,60 \%$. Os principais fatores de risco foram presença de lesões por pressão na

Scielo Cavalcanti Lesão por pressão relacionada a dispositivos médi2018(5) cos: frequência e fatores associados. admissão, com associação significativa para a formação da LPRDM , e pacientes que evoluíam ao desfecho óbito, com associação para formação de LPRDM; não houve associação significativa entre o fator de risco, tempo de internação e local da LPRDM.

\begin{tabular}{|c|c|c|c|}
\hline Scielo & Torres 2016(1) & $\begin{array}{c}\text { Incidência de Úlceras por Pressão Associadas a } \\
\text { Dispositivos Médicos }\end{array}$ & $\begin{array}{l}\text { Os fatores que influenciam a incidência destas lesões são relacio- } \\
\text { nados à condição clínica da pessoa em situação crítica. Enfermeiros } \\
\text { desempenham um papel importante na prevenção das lesões por } \\
\text { pressão e úlceras por pressão associadas a dispositivos médicos e } \\
\text { também no seio da equipe multidisciplinar nos cuidados à pessoa. } \\
\text { Idosos são mais suscetíveis ao desenvolvimento de tais feridas. }\end{array}$ \\
\hline
\end{tabular}

Fonte: Autores, 2021 
de prevenção, reduzem a ocorrência de LPRDM. A capacitação da equipe é essencial para ações de cuidados de prevenção e tratamento visto que estudos apontaram que $90 \%$ da equipe participante, tem dificuldades na classificação normativa das lesões por pressão, interferindo diretamente na avaliação e desenvolvimento de estratégias de enfrentamento de maneira mais efetiva. Os estudos descrevem que quanto maior o tempo de hospitalização, maior o risco de desenvolver lesões. Em relação aos dispositivos médicos mais propensos a desenvolver uma lesão por pressão estão as máscaras de ventilação não invasiva, tubo endotraqueal, cateter venoso e colar cervical, que aumentam de 2 a 4 vezes as chances de desenvolver lesões por pressão. As áreas mais atingidas são: o nariz, lábios, mãos e regiões occipital e cervical.

\section{DISCUSSÃo}

Os principais fatores de risco para o desenvolvimento de LPPRDM são: hipotensão, instabilidade hemodinâmica, comorbidades, histórico de lesões por pressão, edema generalizado, histórico de tabagismo ${ }^{(11,12)}$.

Os atributos físicos dos dispositivos médicos, como a característica do seu material e estrutura física, podem induzir a ocorrência de LPRDM. Quanto menos elástico ou mais duro o dispositivo, maior o risco. A facilidade de ter fricção e pressão exercida sobre o tecido é maior quando o dispositivo está duro e também se a estrutura física do dispositivo facilita a pressão direta na pele do paciente, assim como também foi observado que o tempo de permanência, paciente críticos ou aqueles que requerem necessidade de qualquer tipo de DM, todos esses estão mais propensos a ocorrência dessas lesões. Diversos dispositivos médicos estão ligados às lesões, tendo em vista que os que mais se destacam são: máscaras de

ventilação não invasiva, tubo orotraqueal, dispositivos para traqueostomia, catéter nasogástrico, catéter de gastrostomía, colar cervical, cateter vesical de demora, cateter instalado na artéria radial e tala imobilizadora ${ }^{(12,13)}$.

Para desenvolver um cuidado de qualidade com as LPRDM, é necessário que tenha alguns princípios para

\section{Os principais} fatores de risco para o desenvolvimento de LPPRDM são: hipotensão, instabilidade hemodinâmica, comorbidades, histórico de lesões por pressão, edema generalizado, histórico de tabagismo

eficácia desses cuidados, assim como inspeção da pele, realocação dos dispositivos e que toda a equipe multiprofissional tenha conhecimento sobre as lesões desde o desenvolvimento e quais suas causas. Deste modo os dispositivos médicos não podem ser classificados como inofensivos ao ter participação para o desenvolvimento de
LPP, principalmente se for uma lesão de espessura total. Contudo os dispositivos médicos e de fixação são fundamentais, os cuidados da equipe de enfermagem deve ser primordial, tem que ser analisado o ajuste adequado, a necessidade de permanência, a segurança desses dispositivos médicos, e também a realização cabível de estratégias de prevenção.

São apontados como recomendações relevantes para a prevenção de (LPs), a avaliação de risco e cuidados preventivos da pele e dos tecidos, terapias que resultam na prevenção dessas lesões, nutrição, reposicionamento e mobilização no leito, superfícies de apoio e cuidados com dispositivos para cuidado em saúde. O uso de escalas para identificar o risco e a predisposição do paciente acometido por LP está entre as mais importantes medidas preventivas. Essas medidas contribuem para a elaboração de um plano de cuidados favorável, impedindo ou minimizando o crescimento das lesões ${ }^{(14)}$.

Falando em cuidado o enfermeiro é o profissional que tem maior proximidade com os pacientes, porquanto a preservação da inteireza da pele está inserida na estratégia de cuidado da enfermagem que por sua vez, precisa identificar o risco do aparecimento e crescimento de lesões, para trabalhar no cuidado e na prevenção na integridade da pele atingida. A probabilidade de o paciente desenvolver lesão por pressão é analisada por meio da escala de avaliação de risco, na qual geram escores para esse fim, o meio de classificação altera entre as escalas. Atuações e providências que vão ser utilizadas posteriormente a classificação de risco do paciente, necessitam ser precisas com o intuito de que toda a equipe esteja apta a lidar com situações de alto e baixo risco para o aparecimento de lesão por pressão, visando a prevenção de forma prévia e eficaz ${ }^{(14)}$.

Os estudos analisados propõem como principais precauções preven- 
tivas para o aparecimento de lesões: evitar o posicionamento sobre áreas corporais avermelhadas apontadas com risco elevado, preservação constante de pele limpa e seca por meio de produtos de $\mathrm{pH}$ estável como da pele; não realizar fricção nas regiões com ruborização; para pacientes com problemas de incontinência, a higienização deverá ser realizada momentaneamente a cada ocorrência e a pele precisa ser protegida da exposição à umidade diminuindo as danificações, o uso de hidratante ajuda a diminuir esses danos, se faz necessário a criação de plano de cuidados individuais $^{(14)}$

Analisando esse contexto, pode se observar que essas condutas são adotadas no cuidado aos pacientes, porém podem ser direcionadas também aos profissionais da saúde que faz o uso de máscaras respiratórias e outros EPIs, com o intuito de proteger a pele desses profissionais que estão expostos a diversos fluidos corpóreos. Medidas como proteger a pele, com uma cobertura preventiva como interface entre a pele e a área de fixação da máscara, especialmente se o uso for prolongado a exemplo espuma de poliuretano fina, silicone, filme transparente ou placas de hidrocolóide extrafino; Traçar momentos de desopressão, remoção da máscara a cada duas horas; observar constantemente a pele os sinais de dor, incômodo, vermelhidão e lesões; Em áreas já lesionadas com rubor, eczema, abster-se da colocação de máscaras e EPIs, é necessário antes cuidados tópi- $\cos ^{(15)}$.

A combinação do discernimento clínico, juntamente com as escalas de avaliação do risco para LP ajudam a auxiliar os profissionais a instituir a intervenção mais apropriada para o paciente. A avaliação em pacientes deverá acontecer assim que forem admitidos na UTI, a periodicidade deverá ser refeita logo que for oportuno, e quando as alterações se fizerem presentes, e ou se o estado de saúde do paciente se agravar. Umas das escalas mais usadas a nível mundial é a Escala de Braden foi aprovada no Brasil, para a língua portuguesa, e tem seu uso publicado em contextos, da prática e pesquisas ${ }^{(16)}$.

A atenção em relação a essas escalas é primordial, pois viabiliza o progresso de uma abordagem estruturada, individualizada e sistematizada para solucionar as dificuldades achadas e alargar os aspectos simplificadores, relacionado ao cuidado de enfermagem voltado à prevenção de lesões por pressão, visto que a eficácia do cuidado de enfermagem está profundamente relacionada a uma menor ocorrência de lesões nas instituições que desempenham assistência à saúde ${ }^{(14)}$.

\section{CONCLUSÃO}

Os principais fatores de risco para o paciente para o desenvolvimento de LPPRDM são: hipotensão, instabilidade hemodinâmica, comorbidades, histórico de lesões por pressão, edema generalizado, histórico de tabagismo. Em relação aos atributos físicos dos dispositivos médicos, estão o material e flexibilidade do dispositivo, que quanto mais duro, maior o risco. Os dispositivos médicos que causam a maioria das LPPRDM são: máscaras de ventilação não invasiva, tubo orotraqueal, colar cervical, cateter venoso. As medidas que devem ser adotadas para a prevenção são: o alívio da pressão, a escolha adequada de materiais e a fixação adequada dos mesmos. É essencial que os profissionais de enfermagem tenham o conhecimento acerca do uso adequado destes dispositivos para que realizem a adequada avaliação de risco com uso de escalas e cuidados preventivos da pele e dos tecidos, terapias que resultam na prevenção dessas lesões, nutrição, reposicionamento e mobilização no leito, superfícies de apoio e cuidados com dispositivos para cuidado em saúde.

Este estudo trata-se de um recorte de Trabalho de Conclusão de Curso, onde a busca de literatura ocorreu nos meses de março a maio de 2020. Com o advento da Pandemia de Coronavírus, os autores acreditam que futuros estudos apresentaram mais dispositivos médicos relacionados ao desenvolvimento de lesões por pressão tanto em paciente acometidos pelo COVID-19 devido ao longo período de internação e em profissionais de saúde que ficam paramentados com EPIs por longos período para a prestação de cuidados a estes pacientes.

\section{Referências}

1. Torres RS. Incidência de úlcera por pressão associada a dispositivos médicos. Viana do Castelo: Instituto politécnico de Viana do Castelo- Escola superior de Saúde; 2016.

2. Moraes JT, Borges EL, Lisboa CR, Cordeiro DCO, Rosa EG, Rocha NA. Conceito e classificação de lesão por pressão: Atualização do national pressure ulcer advisory painel. Rev Enferm Cent Oeste Mineiro (RECOM). 2016;6(2):2292-306.

3. Santin Júnior LJ, Carrara GLR, Possidônio PB, Larêdo SMP, Nogueira LDP. Educação permanente: ferramenta de aprimoramento assistencial às lesões por pressão. Rev Enferm UFEP. 2019;13(5):1115- 23.

4. National Pressure ulcer advisory panel. Washington: Pressure ulcer stages revised; 2016.

5. Cavalcanti EO. Lesão por pressão relacionada a dispositivos médicos: Frequência e Fatores Associados. Brasília: Universidade de Brasília; 2018.

6. Black JM, Cuddigan JE, Walko MA, Didier LA, Lander MJ, Kelpe MR. Medical device related pressure ulcers in hospitalized patients. Int Wound J. 2010;7(5):358-65. 


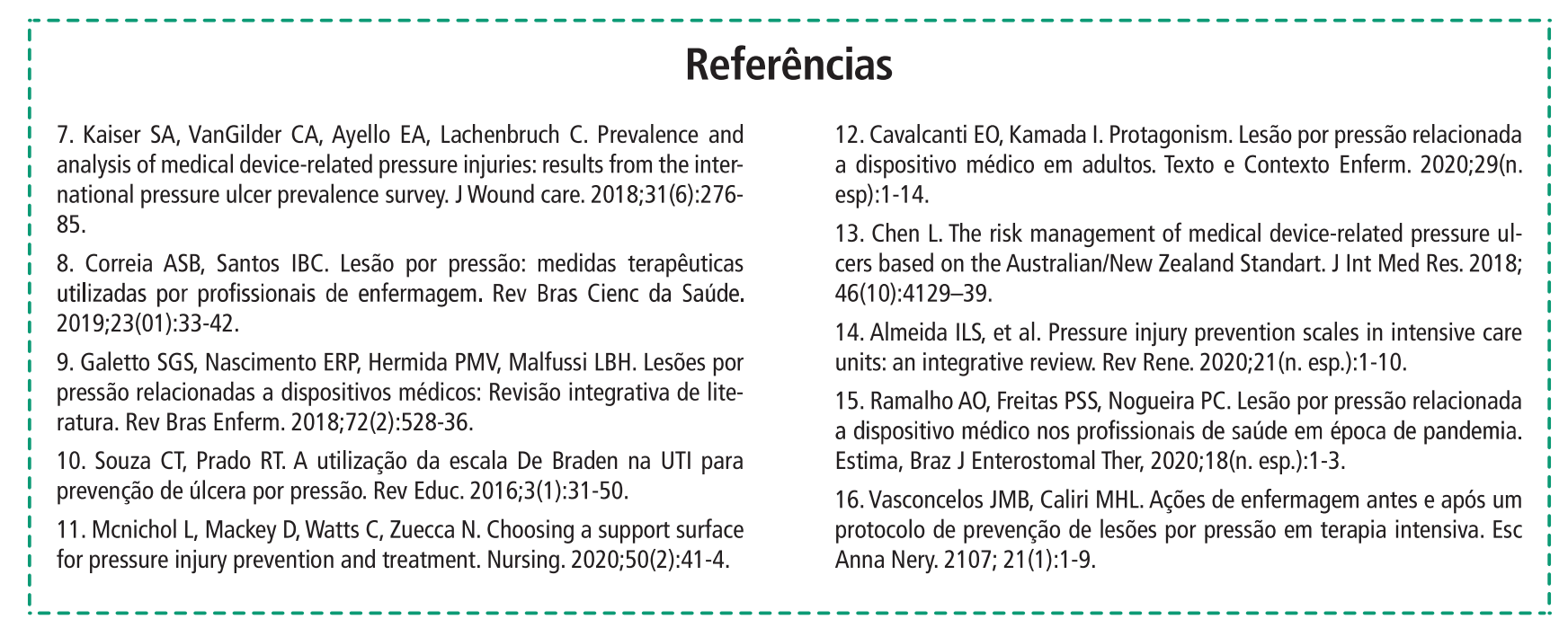

